
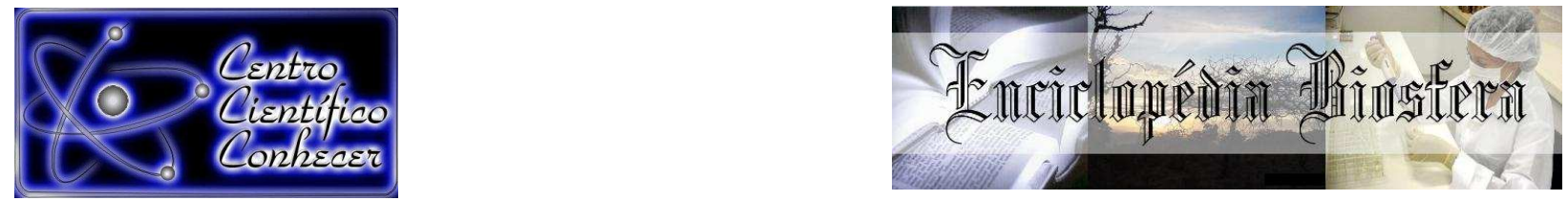

\title{
CARACTERIZAÇÃO DO PERFIL DE DEPENDENTES DE ÁLCOOL: RELEVÂNCIA PARA O ENTENDIMENTO DA ORIGEM DO VÍCIO
}

Patrícia Bossolani Charlo Sanches ${ }^{1}$, Elizangela dos Anjos Silva ${ }^{2}$, Victor Augusto Pacheco $^{3}$, Amanda Platkitka Maximiano $^{3}$, Marcelo Picinin Bernuci ${ }^{2,4}$

${ }^{1}$ Mestranda do Programa Mestrado em Promoção da Saúde do Centro Universitário de Maringá - UNICESUMAR, Av. Guedner, 1610, Maringá-PR, 87050-900, Brasil email: (patbcs@hotmail.com)

${ }^{2}$ Docente do Curso de Medicina do Centro Universitário de Maringá - UNICESUMAR,

Av. Guedner, 1610, Maringá-PR, 87050-900 Brasil

${ }^{3}$ Acadêmico do Curso de Medicina do Centro Universitário de Maringá UNICESUMAR, Av. Guedner, 1610, Maringá-PR, 87050-900 Brasil

${ }^{4}$ Docente do Programa Mestrado em Promoção da Saúde do Centro Universitário de Maringá - UNICESUMAR, Av. Guedner, 1610, Maringá-PR, 87050-900 Brasil

Recebido em: 08/09/2015 - Aprovado em: 14/11/2015 - Publicado em: 01/12/2015 DOI: http://dx.doi.org/10.18677/Enciclopedia_Biosfera_2015_038

O consumo de álcool aumentou significativamente nos últimos anos e se tornou um problema de saúde pública. Enquanto ainda não haja um consenso quanto à origem do vício, estudos destinados a caracterização do perfil dos alcoólatras e de peculiaridades cotidianas possivelmente relacionadas com o vício podem auxiliar 0 desenvolvimento de políticas públicas direcionadas ao controle do alcoolismo. Assim o objetivo do presente estudo foi delinear e caracterizar o perfil dos alcoólatras atendidos no Centro de Atenção Psicossocial Álcool e Drogas (CAPS AD) do município de Maringá/PR, identificando os possíveis fatores envolvidos com a origem do vício. Foi aplicado um questionário semi-estruturado a todos os pacientes alcóolatras do CAPS AD $(n=42)$, contendo questões direcionadas na compreensão dos fatores sociais, econômicos e psicológicos que levaram o indivíduo ao alcoolismo. Todos os indivíduos alcoólatras atendidos eram do sexo masculino com prevalência de idade entre 30 e 60 anos e de maioria (69\%) branca. Com relação ao estado cívil a maioria (62\%) são solteiros ou separados. Cerca de $90 \%$ dos usuários iniciaram o consumo de álcool antes de completarem 20 anos, e 67\% dos indivíduos revelaram que sua proximidade ao consumo abusivo se deve a influência de amigos e ou familiares. Conclui-se que nesta população específica o início do consumo abusivo de álcool ocorreu precocemente e que questões de convívio social foram relevantes para a origem do vício.

PALAVRAS-CHAVE: Alcoolismo, bebidas alcoólicas, estresse 


\title{
CHARACTERIZATION OF THE ALCOHOL DEPENDENT PROFILE: RELEVANCE FOR UNDERSTANDING THE ORIGIN OF THE ADDICTION
}

\begin{abstract}
Alcohol consumption has increased significantly in recent years and has become a public health problem. While there is not yet a consensus as to the origin of addiction, studies to characterize the profile of alcoholics and everyday peculiarities possibly related to addiction can assist the development of public policies aimed at controlling alcoholism. So the aim of this study was to delineate and characterize the profile of alcoholics attended in Drug and Alcohol Psychosocial Care Center (CAPS $A D$ ) of Maringa city - PR, identifying the possible factors involved in the origin of addiction. A semi-structured questionnaire to all alcoholics patients CAPS AD was applied $(n=42)$, with questions aimed at understanding the social, economic and psychological factors that led the individual to alcoholism. All attended alcoholics individuals were male with the prevalence of ages between 30 and 60 years and most of them (69\%) were white. The majority $(62 \%)$ were single or separate. About $90 \%$ of users began drinking alcohol before reaching 20 years, and $67 \%$ of subjects revealed that its proximity to the abuse was due to the influence of friends and or family. Conclude that in this particular population the alcohol abuse occurs early in life and social life issues were relevant to the origins of addiction.
\end{abstract}

KEYWORDS: Alcoholism, alcoholic beverages, stress

\section{INTRODUÇÃO}

O consumo nocivo do álcool provoca cerca de 3,3 milhões de mortes por ano, o que compreende cerca de $6 \%$ de todas as mortes, e contribui com $5 \%$ da carga global de doenças de acordo com o último relato da Organização Mundial de Saúde (OMS) sobre o estado global da relação entre álcool e saúde (OMS, 2014). No Brasil, ainda segundo esta instituição, apenas no ano de 2012, mais de $60 \%$ das mortes por doenças hepáticas foram associadas ao consumo de álcool, e cerca de $20 \%$ das mortes por acidentes de trânsito foram atribuídas ao álcool.

Embora a dependência alcoólica esteja associada a fatores ambientais, sociais e genéticos (LEVEY et al., 2014; WIERS et al., 2014), ainda não há um consenso quanto cada um destes fatores contribui para o desenvolvimento do vício. Há evidências que sugerem, que a origem do consumo nocivo pelo álcool é aprendida e fortemente influenciada por aspectos culturais e sociais, são as chamadas teorias cognitivas, psicológicas ou socioculturais (PECHANSKY et al., 2014); ou ainda evidências que apontam que a herança genética é a principal responsável pelo desenvolvimento do vício, denominada teoria biológica (LEVEY et al., 2014; RODRIGUES, et al., 2014). Há também, relatos de que em muitos casos o consumo abusivo de álcool está associado à fuga de algum sofrimento mental ou emocional, interligado com o ambiente profissional ou pessoal (HERMANSSON et al., 2010; BRITES \& ABREU, 2014).

Independente da contribuição de cada teoria para a explicação da origem do vício, o conhecimento acerca dos motivos que fazem do indivíduo um consumidor crônico do álcool, é sem dúvida fator preponderante para o delineamento de ações mais eficazes de combate ao vício. Dessa forma, o presente estudo tem o objetivo de delinear o perfil dos alcoólatras de um Centro de Atenção Psicossocial Álcool e Drogas (CAPS AD) e identificar os motivos que influenciaram o início do consumo nocivo de álcool. 


\section{MATERIAL E MÉTODOS}

Trata-se de um estudo descritivo utilizando-se da aplicação do questionário estruturados CAGE validado no Brasil desde 1983, respeitando os princípios éticos em pesquisa clínica descrevendo os possíveis fatores relacionados com a origem do vício e questões para obtenção de dados sociodemográficos, direcionados aos dependentes de álcool acompanhados pelo CAPS AD $(n=44)$ do Município de Maringá-PR cujo protocolo de pesquisa foi aprovado pelo Comitê de Ética em Pesquisa do Centro Universitário de Maringá - CESUMAR, sob o número 1.134.937 e o Termo de Consentimento Livre e Esclarecido (TCLE) assinado por todos os sujeitos da pesquisa.

Foram incluídos na pesquisa apenas os indivíduos inscritos no processo de recuperação e tratamento do CAPS AD no período de Julho de 2015 e que assinaram o TCLE. Foram excluídos todos os usuários em que a queixa principal de tratamento não estava relacionado ao consumo de álcool, bem como os que não preencheram corretamente os questionários. Após aplicação dos critérios, a amostra do presente estudo foi composta de 42 dependentes alcoólicos.

O questionário utilizado para coleta de dados foi aplicado individualmente logo após o término das reuniões de grupo com a equipe multiprofissional que envolviam profissionais da enfermagem, psicólogos, terapeutas ocupacionais e médicos. Os dados coletados foram analisados por meio de estatística descritiva das variáveis, tratados pelo programa TabWin, versão 3.6 e desenvolvidos por meio de planilhas do Excel.

\section{RESULTADOS E DISCUSSÃo}

Detectou-se que $100 \%$ dos pacientes são do sexo masculino com prevalência de idade entre 30 a 60 anos e em sua maioria (69\%) de cor de pele branca. Quanto ao estado civil, apenas $38 \%$ dos pacientes mantinham relação de união estável com um cônjuge e $62 \%$ deles não mantinham. Embora a maioria dos pacientes possuísse algum grau de instrução, menos de 10\% deles tinham ensino superior e em sua maioria $(61,9 \%)$ declaram ter renda familiar entre um e cinco salários mínimos. Quanto à atividade profissional, $50 \%$ dos pacientes responderam estar em plena atividade enquanto que a outra metade respondeu estar aposentado (26\%); inativos (17\%) e afastados (7\%). Os dados sociodemográficos dos pacientes alcoólatras do CAPS AD do Município de Maringá estão representados na tabela 1.

Embora o consumo de álcool por mulheres tenha aumentado expressivamente ao longo das últimas décadas, os homens ainda são os principais consumidores, atingindo cerca de 22 litros de álcool puro por ano contra apenas cerca de 9 litros pelas mulheres (OMS, 2014). De acordo com um estudo realizado por MOURA \& MALTA (2011), a principal característica sócio-demográfica relacionada com o consumo de álcool é o grau de escolaridade, que geralmente representa uma relação inversa, ou seja, quanto menor a escolaridade maior o consumo. Porém, o uso abusivo não é exclusivos aqueles com menor instrução, podendo ser encontrado em níveis de escolaridade mais elevados.

Outra associação relevante tem sido feita entre renda familiar e consumo de álcool, em que indivíduos com reduzido poder aquisitivo estão mais vulneráveis ao vício pelo álcool (GALDURÓZ et al., 2010; PINSKY, et al., 2010; ROZIN \& ZAGONEL, 2012). Este fato tem sido relacionado ao contexto de que a classe social baixa tem menor interação com atividades sociais e condições financeiras para arcar com os custos do vício. Contradizendo outros estudos internacional, que relacionam a classe social baixa com maiores índices de consumo, facilitada pela proximidade 
aos fornecedores ou ao convívio social (CARLINI-COTRIM, et al., 2000; BAUS, et al., 2002; RAMÍREZ; ANDRADE, 2005; MARTINO, et al., 2008). Na população em estudo, tanto os fatores educacionais quanto financeiros parecem não ser preponderantes para o vício, visto que a maioria dos pacientes apresentou algum grau de instrução e renda familiar entre 1 a 5 salários mínimos. Por outro lado, a maioria deles não mantinha relação de união estável com um conjugue o que segundo MOURA \& MALTA (2011) é um fator importante para o aumento da incidência do alcoolismo, pois a relação estável oferece ao conjugue um elo de compromisso e um apoio psicológico em vários momentos da vida do indivíduo.

TABELA 1- Caracterização do perfil sociodemográfico dos pacientes do CAPS AD de Maringá-PR.

\begin{tabular}{|c|c|c|}
\hline Variáveis & $\begin{array}{c}\text { Frequência } \\
\text { absoluta (FA) }\end{array}$ & $\begin{array}{c}\text { Frequência } \\
\text { relativa (FR) \% }\end{array}$ \\
\hline \multicolumn{3}{|l|}{ Idade } \\
\hline $0-20$ & 1 & 2,38 \\
\hline $21-30$ & 6 & 14,29 \\
\hline $31-40$ & 10 & 23,81 \\
\hline $41-50$ & 13 & 30,95 \\
\hline $51-60$ & 9 & 21,43 \\
\hline$>61$ & 3 & 7,14 \\
\hline \multicolumn{3}{|l|}{ Classificação Racial } \\
\hline Branco & 29 & 69,05 \\
\hline Negro & 7 & 16,67 \\
\hline Mestiço/mulato & 6 & 14,29 \\
\hline Asiático & 0 & 0,00 \\
\hline \multicolumn{3}{|l|}{ Estado civil } \\
\hline Casado/Amasiado & 16 & 38,10 \\
\hline Solteiro/Separado & 26 & 61,90 \\
\hline Viúvo & 0 & 0,00 \\
\hline \multicolumn{3}{|l|}{ Grau de Escolaridade } \\
\hline Nenhuma & 1 & 2,38 \\
\hline Básico & 10 & 23,81 \\
\hline Fundamental & 13 & 30,95 \\
\hline Médio & 14 & 33,33 \\
\hline Superior & 4 & 9,52 \\
\hline \multicolumn{3}{|l|}{ Atividade profissional } \\
\hline Plena Atividade & 21 & 50,00 \\
\hline Aposentados & 11 & 26,19 \\
\hline Inativos & 7 & 16,67 \\
\hline Afastados (Auxílio Doença) & 3 & 7,14 \\
\hline \multicolumn{3}{|l|}{ Renda mensal familiar } \\
\hline Até 1 salário mínimo & 15 & 35,71 \\
\hline Entre 1 e 5 salários mínimos & 26 & 61,90 \\
\hline Entre 5 e 10 salários mínimos & 1 & 2,38 \\
\hline Mais de 10 salários mínimos & 0 & 0,00 \\
\hline
\end{tabular}

Os dados referentes à caracterização do consumo de álcool pelos pacientes do CAPS AD estão apresentados na tabela 2. Nota-se que cerca de $90 \%$ dos usuários iniciaram o consumo de álcool precocemente, ressaltando em que um deles relatou o início aos sete anos de idade. Quanto ao início do consumo abusivo $48 \%$ declararam aumento do consumo até os 20 anos, e mais da metade $(52 \%)$ dos indivíduos, ingerem no mínimo seis copos de bebida alcoólica diariamente. Com 
relação ao fator que contribui ou contribuiu para a aproximação do álcool, cerca de $67 \%$ dos indivíduos revelaram que sua proximidade esteve relacionada com a influência de amigos e/ou familiares. Quanto ao familiar mais próximo com consumo abusivo de álcool, cerca de $31 \%$ dos indivíduos referiram a figura paterna.

Quanto mais precoce o contato inicial dos jovens com o álcool maior a propensão do aumento dos casos de dependência alcoólica (LARANJEIRA, et al., 2009; ROSSI et al., 2012). A precocidade na experimentação do álcool verificada neste estudo, também foi observada em outros estudos no Brasil, que apontam que cerca de 60 a $90 \%$ dos adolescentes que cursam o ensino médio, após o seu término, já consumiram álcool, comumente em festas, oferecidas e consumidas por amigos e ou familiares, tornando exceção a abstinência total dentro dessa faixa etária (STICKLEY, et al., 2013; REIS \& OLIVEIRA, 2015). Segundo VIERA \& colaboradores (2007), em seu estudo sobre a evidência da associação entre o uso precoce de álcool, a incidência do primeiro contato com as bebidas alcoólicas entre os jovens chegará aproximadamente até o ano de 2020 em cerca de $25 \%$, e esse início precoce acontecerá antes desses jovens completarem dez anos de vida.

TABELA 2- Caracterização do consumo de álcool pelos pacientes do CAPS AD de Maringá-PR

\begin{tabular}{|c|c|c|}
\hline Variáveis & $\begin{array}{c}\text { Frequência } \\
\text { absoluta (FA) }\end{array}$ & $\begin{array}{c}\text { Frequência } \\
\text { relativa (FR) \% }\end{array}$ \\
\hline \multicolumn{3}{|l|}{ Idade de início do consumo } \\
\hline $0-20$ & 38 & 90,48 \\
\hline $21-30$ & 1 & 2,38 \\
\hline $31-40$ & 1 & 2,38 \\
\hline $41-60$ & 2 & 4,76 \\
\hline \multicolumn{3}{|l|}{ Idade do aumento do consumo } \\
\hline $0-20$ & 20 & 47,62 \\
\hline $21-30$ & 12 & 28,57 \\
\hline $31-40$ & 5 & 11,90 \\
\hline $41-60$ & 4 & 9,52 \\
\hline Nunca & 1 & 2,38 \\
\hline \multicolumn{3}{|l|}{ Quantidade de copos ingeridos/dia } \\
\hline Nenhum & 0 & 0,00 \\
\hline $1-3$ & 11 & 26,19 \\
\hline $4-5$ & 9 & 21,43 \\
\hline$>6$ & 22 & 52,38 \\
\hline \multicolumn{3}{|c|}{$\begin{array}{c}\text { Fator que contribuiu para aproximação } \\
\text { do álcool }\end{array}$} \\
\hline Influência amigos/familiares & 28 & 66,67 \\
\hline Problemas pessoal e amoroso & 7 & 16,67 \\
\hline Curiosidade & 4 & 9,52 \\
\hline Depressão & 2 & 4,76 \\
\hline Futebol & $\overline{1}$ & 2,38 \\
\hline \multicolumn{3}{|c|}{ Parente próximo com uso abusivo álcool } \\
\hline Pai & 13 & 30,95 \\
\hline Mãe & 5 & 11,90 \\
\hline Irmão (ã) & 8 & 19,05 \\
\hline Tio & 5 & 11,90 \\
\hline Avôs & 3 & 7,14 \\
\hline Nenhum & 8 & 19,05 \\
\hline
\end{tabular}


Frequentemente, o álcool é oferecido e consumido como meio de interação e socialização (FERREIRA \& TORGAL, 2010; WILLHELM et al., 2015). Esta afirmação foi comprovada quando os participantes do presente estudo foram questionados sobre o significado do álcool, em que cerca de $38 \%$ afirmaram significar um meio de relaxamento ou de bem estar, enquanto que, $30 \%$ referem que o álcool é um motivo para estar com os amigos, $21 \%$ sentiam prazer ao consumir a bebida alcoólica e somente $4 \%$ revelaram ser uma fuga da sociedade. Em concordância com o presente estudo pesquisas descrevem os principais motivos de consumo de bebidas alcoólicas, principalmente relacionados ao convívio com alcoólatras, pois quanto maior o convívio com pessoas que consomem álcool, maior será o fator que predispõe o início ao consumo (FERREIRA \& TORGAL, 2010; STICKLEY et al., 2013; WILLHELM et al., 2015).

De fato, a relação do grau de parentesco com o uso do álcool tem sido amplamente investigada, e sugere-se que quando os usuários têm familiares (pais e irmãos) que consomem álcool dentro do domicílio, esse consumo é visto como algo natural, facilitando a permissão para o início do consumo dos filhos (STOOLMILLER et al., 2012; HAUGLAND et al., 2013; MOHANAN et al., 2014). Assim é imprescindível que novas pesquisas sejam voltadas para estudar o âmbito familiar dos indivíduos que fazem o uso nocivo de bebidas alcoólicas, com o intuito de promover a saúde familiar e evitar que outros membros da família ingressem nesse vício, colaborando assim com iniciativas de implementações de ações de promoção da saúde, possibilitando novas oportunidades de entendimento sobre a origem do vício do álcool. O presente estudo possibilita reflexões sobre o delineamento de novas estratégias de controle do alcoolismo direcionadas principalmente para a população jovem e que considere o potencial do convívio social para o origem do vício pelo álcool.

\section{CONCLUSÃO}

O vício do álcool acomete indivíduos em plena atividade de trabalho, acarretando assim um déficit na capacidade deste exercer sua função no emprego, seja aumentando os índices de absenteísmo ou causando danos físicos e mentais no trabalhador. $O$ início do consumo ocorre de maneira precoce e influenciado principalmente por amigos ou familiares.

\section{REFERÊNCIAS}

BAUS, J.; KUPEK, E.; PIRES, M. Prevalência e fatores de risco relacionados ao uso de drogas entre escolares. Revista Saúde Pública, v. 36, n. 1, p. 40-46, 2002.

BRITES, R. M. R.; ABREU, Â. M. M. Alcohol consumption pattern among workers and socioeconomic profile. Acta Paulista Enfermagem, v. 27, n. 2, p. 93-99, 2014.

CARLINI-COTRIM, B.; GAZAL-CARVALHO, C.; GOUVEIA, N. Comportamento de saúde entre jovens estudantes das redes pública e privada da área metropolitana do Estado de São Paulo. Revista Saúde Pública, v. 34, n. 6, p. 636-645, 2000.

FERREIRA, M. M.; TORGAL, M. C. Tobacco and alcohol consumption among adolescents. Revista Latino Americana de Enfermagem, v. 18, n. 2, p. 255-261, 2010. 
GALDURÓZ, J. C. F.; SANCHEZ, Z. M.; OPALEYE, E. S.; NOTO, A. R.; FONSECA, A. M.; GOMES, P. L. S. Fatores associados ao uso pesado de álcool entre estudantes das capitais brasileiras. Revista Saúde Pública: São Paulo, v. 44, n. 2, p. 267-273, 2010.

HAUGLAND, S. H.; HOLMEN, T. L.; RAVNDAL, E.; BRATBERG, G. H. Parental alcohol misuse and hazardous drinking among offspring in a general teenage population: gender-specific findings from the Young-HUNT 3 study. Biomed Central: Public Health, v. 13, p. 1140, 2013.

HERMANSSON, U.; HELANDER, A.; BRANDT, L.; HUSS, A.; RÖNNBERG, S. Screening and brief intervention for risky alcohol consumption in the workplace: results of a 1-year randomized controlled study. Alcohol Alcohol, v. 45, n. 3, p. 252257, 2010.

LARANJEIRA, R.; PINSKY, I.; SANCHES, M.; ZALESKI, M.; CAETANO, R. Alcohol use patterns among Brazilian adults. Revista Brasileira de Psiquiatria, v. 32, n. 3, p.231-241, 2009.

LEVEY, D. F.; NICULESCU, H. LE.; FRANK, J.; AYALEW, M.; JAIN, N.; KIRLIN, B.; LEARMAN, R.; WINIGER, E.; RODD, Z.; SHEKHAR, A.; SCHORK, N.; KIEFE, F.; WODARZ, N.; MYHSOK-MULLER, B.; DAHMEN, N.; GESGA, C.; NOTHEN, M.; SHERVA, R.; FARRER, L.; SMITH, A. H.; KRANZLER, H. R.; RIETSCHEL, M.; GELERNTER, J.; NICULESCU, A. B. Genetic risk prediction and neurobiological understanding of alcoholism. TransI Psychiatry, v. 20, n. 4, p.391-407, 2014.

MARTINO, S. C.; ELLICKSON, P. L.; MCCAFFREY, D. F. Developmental trajectories of substance use from early to late adolescence: a comparison of rural and urban youth. J Stud Alcohol Drugs, v. 69, n. 3, p. 430-440, 2008.

MOHANAN, P.; SWAIN, S.; SANAH, N.; SHARMA, V.; GHOSH, D. A Study on the Prevalence of Alcohol Consumption, Tobacco Use and Sexual Behaviour among Adolescents in Urban Areas of the Udupi District, Karnataka, India. Sultan Qaboos Univ Med J, v. 14, n. 1, p. 104-112, 2014.

MOURA, E. C.; MALTA, D. C. Alcoholic beverage consumption among adults: sociodemographic characteristics and trends. Revista Brasileira Epidemiologia, v. 14, n. 1, p. 61-70, 2011.

OMS - Organização Mundial da Saúde. Global status report on alcohol and health. 2014.

PINSKY, I.; SANCHES, M.; ZALESKI, M.; LARANJEIRA, R.; CAETANO, R. Patterns of alcohol use among Brazilian adolescents. Revista Brasileira Psiquiatria, v. 32, n. 3, p. 242-249, 2010.

PECHANSKY, F.; SZOBOT, C. M.; SCIVOLETTO S. Uso de álcool entre adolescentes: conceitos, características epidemiológicas e fatores etiopatogênicos. Revista Brasileira Psiquiatria, v. 24, p. 14-17, 2014. 
RAMíREZ, R. M.; ANDRADE, D. La familia y los factores de riesgo relacionados con el consumo de alcohol y tabaco em los niños y adolescentes (Guayaquil-Ecuador). Revista Latino Americana de Enfermagem, v. 13, n. especial, p. 813-818, 2005.

REIS, T. G.; OLIVEIRA, L. C. M. Pattern of alcohol consumption and associated factors among adolescents students of public schools in an inner city in Brazil. Revista Brasileira de Epidemiologia, v. 18, n. 1, p. 13-24, 2015

RODRIGUES, P. F. S.; SALVADOR, A. C. F.; LOURENCO, I. C.; SANTOS, L. R. Padrões de consumo de álcool em estudantes da Universidade de Aveiro: Relação com comportamentos de risco e stress. Análise Psicológica, v. 32, n. 4, p. 453-466, 2014.

ROSSI, J. A. P.; SANTIAGO, K. B.; MARTINS, O. A. Study of fetal alcohol syndrome. Revista Eletrônica de Educação e Ciência, v. 2, n. 1, p. 1-9, 2012.

ROZIN, L.; ZAGONEL, I. P. S. Risk factors for alcohol dependence in adolescents. Acta Paulista Enfermagem, v. 25, n. 2, p. 314-318, 2012.

STICKLEY, A.; KOYANAGI, A.; KOPOSOV, R.; MCKEE, M.; ROBERTS, B.; MURPHY, A. Binge drinking among adolescents in Russia: prevalence, risk and protective factors. Addict Behav, v. 38, n. 4, p. 1988-1995, 2013.

STOOLMILLER, M.; WILLS, T. A.; MCCLURE, A. C.; TANSKI, S. E.; WORTH, K. A.; GERRARD, M. Comparing media and family predictors of alcohol use: a cohort study of US adolescents. BMJ Open, v. 2, p. 543-549, 2012

VIEIRA, D.L.; RIBEIRO, M.; LARANJEIRA, R. Evidence of association between early alcohol use and risk of later problems. Revista Brasileira Psiquiatria, v. 29, n. 3, p. 222-227, 2007.

WIERS, C. E.; STELZEL, C.; PARK, S. Q.; GAWRON, C. K.; LUDWIG, V. U.; GUTWINSKI, S.; HEINZ, A.; LINDENMEYER, J.; WIERS, R. W.; WALTER, H.; BERMPOHL, F. Neural correlates of alcohol-approach bias in alcohol addiction: the spirit is willing but the flesh is weak for spirits. Neuropsychopharmacology, v. 39, n. 3, p. 688-697, 2014.

WILLHELM, A. R.; CABRAL, J. C. C.; STEIGER, J. O.; SILVA, J. F. F.; UGARTE, L. M.; ALMEIDA, R. M. M. Consumo de Álcool na Adolescência e Relação com Uso Excessivo de Bebidas Alcóolicas dos Pais: Estudantes de Quatro Escolas de Porto Alegre. Psico, Porto Alegre, v. 46, n. 2, p. 208-216, 2015. 